\title{
Effect of fiber angle orientation and stacking sequence on mixed mode fracture toughness of carbon fiber reinforced plastics: Numerical and experimental investigations
}

\author{
P. Naghipour ${ }^{\mathrm{a}, \mathrm{b}, *}$, M. Bartsch ${ }^{\mathrm{a}}$, L. Chernova ${ }^{\mathrm{a}}$, J. Hausmann ${ }^{\mathrm{a}}$, H. Voggenreiter ${ }^{\mathrm{a}, \mathrm{b}}$ \\ a Institute of Materials Research, German Aerospace Center (DLR), Linder Hoehe, 51147 Cologne, Germany \\ ${ }^{\mathrm{b}}$ Institute of Aircraft Design (IFB), University of Stuttgart, Pfaffenwaldring 31, 70569 Stuttgart, Germany
}

\section{A R T I C L E I N F O}

\section{Article history:}

Received 13 March 2009

Received in revised form 30 July 2009

Accepted 31 July 2009

Available online $\mathrm{xxx}$

\section{Keywords:}

Mixed mode bending

Multidirectional composites

Cohesive zone modelling

Interface damage simulation

Inter-laminar fracture

\begin{abstract}
A B S T R A C T
This paper focuses on the effect of fiber orientation and stacking sequence on the progressive mixed mode delamination failure in composite laminates using fracture experiments and finite element (FE) simulations. Every laminate is modelled numerically combining damageable layers with defined fiber orientations and cohesive zone interface elements, subjected to mixed mode bending. The numerical simulations are then calibrated and validated through experiments, conducted following standardized mixed mode delamination tests. The numerical model is able to successfully capture the experimentally observed effects of fiber angle orientations and variable stacking sequences on the global load-displacement response and mixed mode inter-laminar fracture toughness of the various laminates. For better understanding of the failure mechanism, fracture surfaces of laminates with different stacking sequences are also studied using scanning electron microscopy (SEM).
\end{abstract}

(C) 2009 Elsevier B.V. All rights reserved.

\section{Introduction}

As laminated composites are widely used in aerospace applications, their failure mechanisms under different loading conditions have been studied extensively by various research groups. Different stacking sequences, fiber orientations and crack propagation directions have considerable effects on the structural response, fracture resistance, and failure mechanisms of the composite laminates. The behaviour of different multidirectional (MD) composite laminates, with different ply orientations, subjected to single or mixed mode delamination has been studied by different research groups [1-8]. As a summarized result of the mentioned references and as explicitly reported in literature [3-5], MD laminates are generally preferred to unidirectional (UD) ones due to their higher interlaminar fracture resistance observed through experiments, which is assumed to be due to extrinsic toughening mechanisms such as blunted crack tips or deviation of the crack from the main crack plane to the adjacent layers and some in-ply energy absorption.

Numerical modelling of mixed mode delamination of MD laminates is a challenging task, since the final failure generally occurs

\footnotetext{
* Corresponding author at: Institute of Materials Research, German Aerospace Center (DLR), Linder Hoehe, 51147 Cologne, Germany. Tel.: +49 2203 6013509; fax: +492203696480.

E-mail address: parya.naghipour@dlr.de (P. Naghipour).
}

as a result of interacting intra-laminar and inter-laminar damage modes. The mathematical models or analytical solutions concerning in-ply (intra-laminar) and out of ply (inter-laminar) damage or fracture are specified in Refs [9-21]. When intra-laminar damage modes such as matrix cracking and fiber rupture are of primary concern, a detailed orthotropic ply damage model must be developed to fully capture the failure mechanism [9-13]. When inter-laminar failure or delamination is the predominant failure mechanism, several methods suggested in literature can be used for simulating interfacial damage initiation and propagation [14-21]. One of the recent appealing techniques used in the numerical modelling of delamination is the cohesive zone approach, which is also applied in this work. Many authors have published papers on the cohesive zone approach or development of interface elements, [16-21], since it was first suggested by Dugdale [16].

The present paper focuses on simulation of mixed mode delamination of composite laminates with different layups and stacking sequences following experimental and numerical approaches. Combination of these two approaches, mainly emphasizing the effect of fiber orientations and stacking sequences on improving the inter-laminar fracture resistance of multidirectional composite laminates with a thermoplastic matrix (PEEK), has not been addressed in detail in literature and is the main scope of this work. The mixed mode bending (MMB) experiments, first suggested by Crews and Reeder [22] are conducted at German Aerospace Centre (DLR). As a result of these experiments the load-displacement 
Table 1

CFRP specimen configurations ( $d$ stands for delamination plane).

\begin{tabular}{ll}
\hline Layups to be considered & Layup name \\
\hline $\begin{array}{l}(+22.5 /-22.5)_{12} \\
+45 /-45 / 0_{3} /-45 / 0 /+45 / 0_{2} /+45 /-45 / \mathrm{d} /+45 /-45 / 0_{2} /+45 / 0 /+45 /\end{array}$ & Layup 22.5 \\
$0_{3} /+45 /-45$ & \\
UD $\left([0]_{24}\right)$ & Layup UD \\
\hline
\end{tabular}

response and inter-laminar fracture mechanisms of different layups under various mode mixities were obtained, and the fracture surfaces were analyzed in detail using SEM. The mixed mode inter-laminar damage is represented by using interface elements, the constitutive mathematical model of which is described in detail in Ref. [20]. Meanwhile, an in-built ply damage model in ABAQUS [23], partially based on the works of Hashin [13] and Matzenmiller et al. [10], is used to define the intra-laminar ply damage behaviour. The numerical model is validated by reproducing the load-displacement response of the conducted MMB experiments and growth of the expected damage.

Following this Section 1, Section 2 describes the material system and the followed MMB experimental procedure. The numerical procedure is summarized in Section 3. Comparisons between numerical and experimental results, the effect of fiber orientations and stacking sequences on the load-displacement response and fracture resistance, and discussions on some SEM samples are all given in Section 4. Finally, a brief summary and conclusions are presented in Section 5.

\section{Experimental program}

\subsection{Test specimens}

Base material used in this study is APC2-prepreg material from Cytec Engineered Materials (Cytec Industries Inc.) consisting of AS4-fibres (60 vol.\%) impregnated with a PEEK matrix. The thickness of each prepreg layer is about $140 \mu \mathrm{m}$. As an initial step, the specified number and orientation of layers (Table 1 ) is stacked together on a heating plate, and a $50 \mathrm{~mm}$ width, $13 \mu \mathrm{m}$ thick polyimide film (Kapton) is placed in the mid-plane of each layup as a delamination starter. In the next step, the completed stacking is equipped with thermocouples and covered with a vacuum bag and textile insulation layers. After applying vacuum, the heating plate is heated up to $400^{\circ} \mathrm{C}$ to melt the PEEK matrix for consolidation. Then the heating is switched off for cooling down. After removing the insulation and vacuum bag a consolidated plate with the desired layup and delamination layer is obtained. Plates with a dimension of about $320 \mathrm{~mm} \times 320 \mathrm{~mm}$ were produced and cut by water jet to final specimen size. Thus, several specimens were produced within one batch. The production of all specimens using the above-mentioned consolidation technique was held at the composite laboratory of German Aerospace Centre (DLR) in Stuttgart. Some known advantages of the thermoplastic polymers such as good formability by reheating and weldability, environmentally friendly processing, and their certification for use in aeronautics were among the main reasons to prefer them to a thermoset matrix material. Each tested specimen was a 24-ply carbon/PEEK laminate, $25 \mathrm{~mm}$ wide, $150 \mathrm{~mm}$ long, and $3.12 \mathrm{~mm}$ thick, and a $13 \mu \mathrm{m}$ polyimide film (Kapton), used as the delamination starter plane.

The coupling stiffness $\left(B_{i j}\right)$ of the chosen laminates is desired to be zero or very close to zero in order to minimize twisting by bending moments and distortion by thermal residual stresses, as the specimens are tested at a temperature different from the consolidation temperature [24]. Meanwhile, stacking sequences, which have equal numbers of $+\theta$ and $-\theta$ plies (balanced layups), are preferred. The specimen stacking sequence should be chosen to minimize and keep a non-dimensional ratio $D_{c}=\left(D_{12}\right)^{2} / D_{11} D_{22}$ smaller than 0.25 in each delamination arm [1,2], where $D_{i j}$ are the elements of the bending stiffness matrix of the sublaminates. Minimization of $D_{c}$ will also minimize the non-uniform toughness value distribution, local mixed mode effects, skewed and curved crack fronts in fracture testing, and the errors in perceived fracture toughness values obtained from experimental load-deflection data. Hence the produced layups have mainly balanced configuration, the value of $D_{c}$ is kept under 0.25 and $B_{i j}$ close to zero, for all of them. The detailed specimen stacking sequences are shown in Table 1.

The 1st layup (layup 22.5) consists of 24 alternating layers with $+22.5^{\circ}$ and $-22.5^{\circ}$ fiber orientations. The whole laminate is symmetric with respect to the laminate mid-plane (subscript $s$ stands for mid-plane symmetry). The 2nd layup (Layup 45) is a 24-ply laminate with $+45^{\circ},-45^{\circ}$, and $0^{\circ}$ fiber orientations (subscripts 3 and 2 show the number of $0^{\circ}$ plies in the layup). The third layup (Layup UD) is a unidirectional 24-ply laminate with $0^{\circ}$ fiber orientations. The delamination plane in all the mentioned layups lies in the middle, between the 12 th and 13 th plies.

\subsection{Experimental procedure and data reduction}

The MMB experiment, first introduced by Crews and Reeder [22], provides the possibility to combine the influence of normal or opening (mode I) and shear or sliding stresses (mode II) on inter-laminar delamination using a single test apparatus shown in Fig. 1. The MMB loading was represented by a superposition of pure mode I and mode II loadings, conducted by a single load $P$. Fig. 1 shows the MMB loading expressed in terms of the applied load $P$, the loading lever length $c$, and the specimen half-span $L$. The relative magnitude of the two resulting loads on the specimen, or in other words the mode mixity, is determined by varying the lever length, $c$. When the applied load is directly above the beam mid-span $(c=0)$, pure mode II is achieved, and pure mode I is applied by removing the beam and pulling up the hinge. The mechanical tests were carried out according to the standardized test for mixed mode bending fracture of fiber reinforced composites [25]. The machine used is a 10 tonnes Instron testing machine equipped with a $100 \mathrm{kN}$ load cell to measure the load for propagating the crack. The load precision is verified to $0.5 \%$ for this load cell. Therefore, the minimum and maximum load levels measured in the experimental set might have an uncertainty ranging from 0.75 to $3 \mathrm{~N}$. Before mounting the MMB test specimen to the testing apparatus, a calibration specimen was used to ensure the accuracy of all measuring equipment. The calibration specimen was a rectangular bar made from steel with a $193 \mathrm{GPa}$ elastic modulus and a flexural rigidity of about $90.12 \mathrm{~N} \mathrm{~m}^{2}$ as suggested in the standard test procedure. Similar to a MMB specimen, tabs were applied to one end, then the MMB apparatus was loaded with the calibration specimen and the load-displacement response was recorded. The slope of this calibration load-displacement curve is then measured to calculate the compliance of the MMB testing system, which must be accurately determined at each setting of lever length, c. After system calibration, the MMB specimen was mounted on the apparatus and in addition to pure mode I and pure mode II tests, experiments with three different mode mixities (30\%, 50\%, and $80 \%$ ) were carried out using the MMB apparatus with a crosshead displacement rate of $0.5 \mathrm{~mm} / \mathrm{min}$ for all the specimens. The loading point displacement and load histories were recorded by using a digital data acquisition system and the optimum data sampling rate was chosen to be $50 \mathrm{~Hz}$. At least three specimens were tested for each mode mixity (i.e., at each mode mix the average of three experiments is taken as the main experimental data), and the differences in the measured data (displacement, loads and propa- 

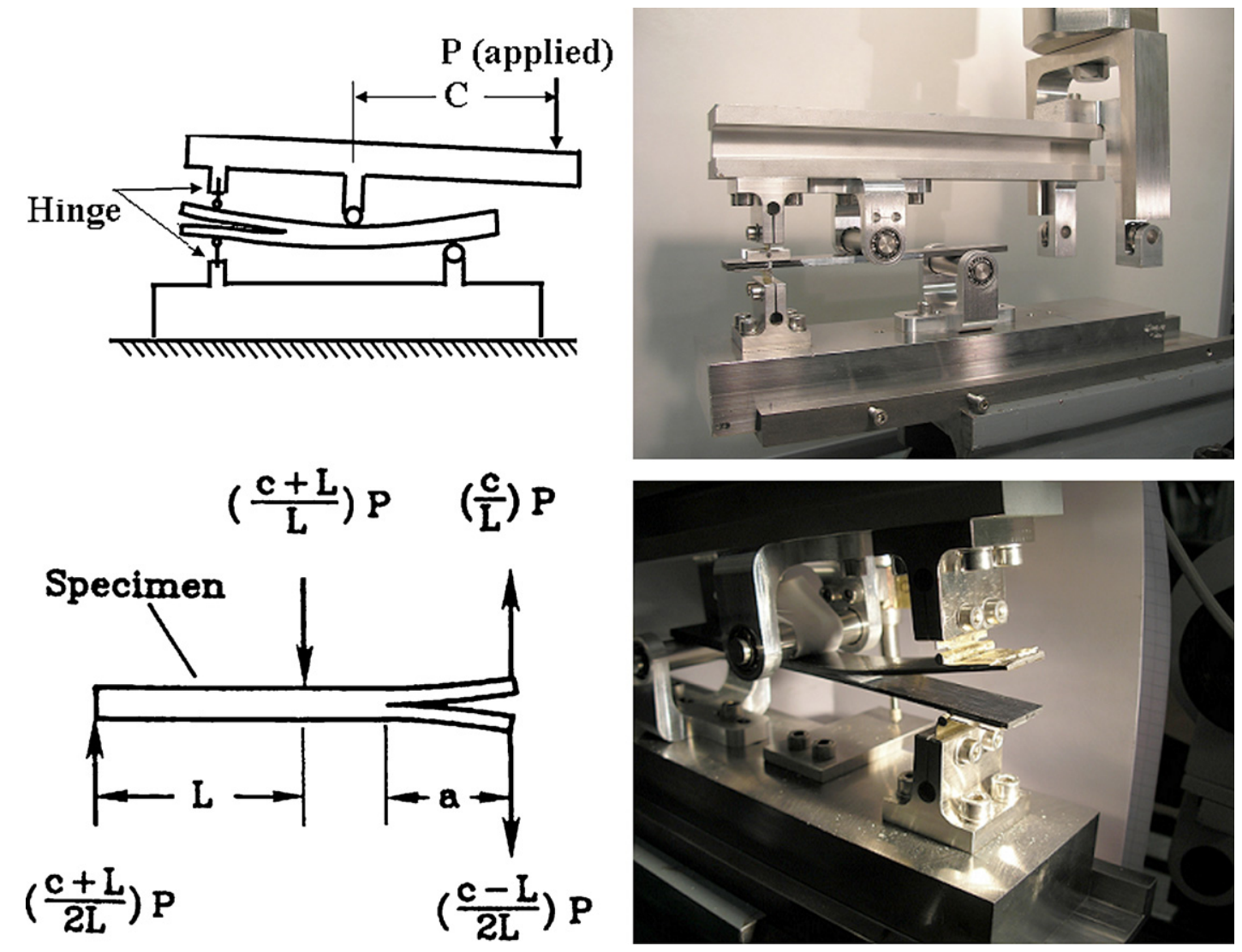

Fig. 1. Apparatus configuration and loading description of MMB test apparatus.

gating crack length) were less than $1.5 \%$. A video camera was placed parallel to the specimen thickness to monitor the crack growth. By using this camera together with a crack event marker, it was possible to determine the load and displacements associated with a specific crack length.

The data reduction procedure to calculate mode I and mode II critical strain energy release rates or fracture toughness $\left(G_{\text {Ic }}\right.$ and $\left.G_{\text {IIC }}\right)$ is explained here. The solutions for mode I and mode II strain energy release rates $\left(G_{I}\right.$ and $\left.G_{I I}\right)$ are derived from "beam theory" solutions, also described in Ref. [26] by Williams for general problems involving delaminated homogeneous materials. The beam theory equations are further "corrected" by energy terms associated with shear deformation, the rotation of arms at the delamination tip, and plastic deformation ahead of the crack tip incorporated as crack tip correction factors, $\chi$ [27-28]. According to this "corrected beam theory", stated in Eq. (1), $G_{I}$ and $G_{I I}$ are mainly functions of applied load $(P)$, loading lever length $(c)$, propagating crack length $(a)$, crack tip correction factor $(\chi)$, and mechanical and geometrical properties of the specimen:

$$
\begin{aligned}
G_{\mathrm{I}} & =\frac{6 P^{2}\left(a+\chi_{n}\right)^{2}(1+\varphi)}{W^{2} h_{u}{ }^{3} E_{11 f, u} \varphi}\left[\frac{(1+2 \varphi) c-L}{2(1+\varphi) L}\right]^{2} \varphi=\frac{E_{11 f, l} h_{l}{ }^{3}}{E_{11 f, u} h_{u}{ }^{3}} \\
G_{\mathrm{II}} & =\frac{3 P^{2}\left(a+\chi_{s}\right)^{2}}{2 W^{2} h_{u}{ }^{3} E_{11 f, u}}\left[\frac{8 h^{3} E_{11 f}-(1+\varphi) h_{u}{ }^{3} E_{11 f, u}}{(1+\varphi) 8 h^{3} E_{11 f}}\right]\left(\frac{L+c}{L}\right)^{2}
\end{aligned}
$$

The crack tip corrections for normal and shear modes, $\chi_{n}$ and $\chi_{s}$, are computed adapting the existing solutions for UD composites. In order to obtain $\chi_{n}$, Williams' elastic foundation solution for the Double Cantilever Beam test [26] is modified to the following form, Eq. (2), which is also used in the ASTM D6671 data reduction pro- cedure:

$$
\chi_{n}=h_{u} \sqrt{\frac{E_{11 f, u}}{11 G_{13}}\left\{3-2(\Gamma / 1+\Gamma)^{2}\right\}} \quad \Gamma=1.18 \frac{\sqrt{E_{11 f, u} E_{22, u}}}{G_{13, u}}
$$

Furthermore according to [29], based on the analysis of End Notched Flexure test, $\chi_{s}$ can be obtained through Eq. (3):

$\chi_{s}=h_{u} \sqrt{\frac{E_{11 f, u}}{72 G_{13, u}}}$

In the above expressions $E_{11 f}$ stands for longitudinal flexural modulus in fiber direction of the half-laminate obtained through Classical Laminate Theory. $E_{22}$ and $G_{13}$ are transverse modulus perpendicular to fiber direction and transverse shear modulus of the whole laminate, respectively. The subscripts $(u)$ and $(l)$ correspond to the upper and lower sublaminates, and $W$ and $h$ are width and halfthickness of the specimen. It is worth mentioning that the crack tip correction factors are assumed to be the same for upper and lower sublaminates here.

The critical value of the applied load in the determination of $G_{I c}$ and $G_{\text {IIc }}$ is chosen as the load corresponding to the $1 \mathrm{st}$ visible nonlinearity in load-displacement curve $\left(P_{N L}\right)$ [30]. The critical delamination length is taken as the propagation crack length marked through the experiment. If the required mechanical properties of a laminate like $\left(E_{11 f}, E_{22}\right.$, and $\left.G_{13}\right)$, geometrical properties (width, thickness, and length) and mixed mode fracture experimental data (Ex: load-displacement and crack propagation data) are available, then one can directly calculate the critical strain energy release rates using above-mentioned Eqs. (1)-(3).

Failure criteria used in damage evolution process is based on strain energy release rate in this work, which seems to be a good measure of a materials resistance to delamination extension, and 


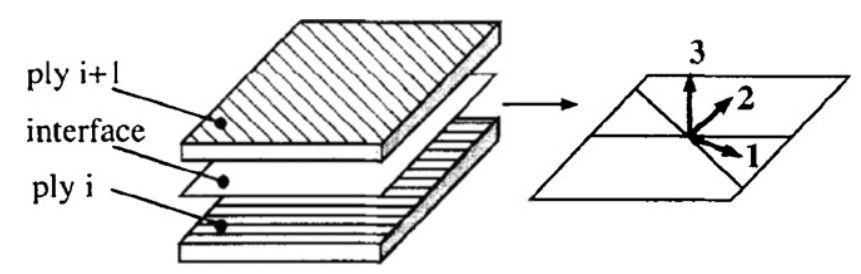

Fig. 2. Schematic of the numerical model (lamina + interface) [12].

most of the suggested failure criteria can be written in terms of critical strain energy release rate or fracture toughness. In this study, in order to accurately account for the variation of fracture toughness as a function of mode ratio in the AS4/PEEK composite, the mixed mode criterion proposed by Benzeggagh and Kenane [31] (Eq. (4)), is used:

$G_{I c}+\left(G_{\text {IIc }}-G_{I c}\right)\left(\frac{G_{\text {shear }}}{G_{T}}\right)^{\eta}=G_{c} \quad G_{T}=G_{I}+G_{\text {shear }}$

$G_{T}$ : Total fracture toughness

According to [31], the mixed mode failure response of the material is described by plotting the total critical fracture toughness $G_{c}$ versus different mode mixities $\left(G_{\text {shear }} / G_{T}\right)$. Parameter $\eta$ in Eq. (4) maintains the shape of the failure locus in the mixed mode plane, and the most accurate failure criterion is the one matching the material response when plotted in this mixed mode diagram.

\section{Numerical simulations}

The numerical model created in ABAQUS [23] consists of individual damageable plies to capture any in-ply damage and interface elements in between them as shown schematically in Fig. 2. The mathematical damage models used for the ply and the interface are described in this section.

\subsection{Ply damage model}

The in-ply damage in each lamina is described using the in-built Hashin damage model in ABAQUS [23]. An orthotropic material inplane stress for each ply is assumed prior to damage initiation. Beginning of degradation of the ply response, or in other words the damage initiation, is due to four main failure criteria, namely: fiber ruptures in tension, fiber buckling in compression, matrix cracking under transverse tension and shearing, and matrix crushing under transverse compression. Damage propagates when the total fracture energy in any of the four mentioned cases reaches its maximum value $\left(G_{c \max }\right)$ specified by the user as an input parameter. During damage propagation, three non-negative in-ply damage parameters, $d_{f}, d_{m}$, and $d_{s}$ reduce the ply stiffness numerically in fiber, transverse, and shear directions, respectively, until the final failure point is reached. Therefore, the degradation of the ply stress tensor $(\boldsymbol{\sigma})$ can be written as [10]

$\boldsymbol{\sigma}=\frac{1}{1-\left(1-d_{f}\right)\left(1-d_{m}\right) v_{12} v_{21}} \mathbf{C}\left[\begin{array}{c}\varepsilon_{11} \\ \varepsilon_{22} \\ \varepsilon_{12}\end{array}\right]$

$\mathbf{C}=\left(\begin{array}{lll}E_{11}\left(1-d_{f}\right) & \left(1-d_{f}\right)\left(1-d_{m}\right) E_{22} v_{21} & 0 \\ \left(1-d_{f}\right)\left(1-d_{m}\right) E_{11} v_{12} & E_{22}\left(1-d_{m}\right) & 0 \\ 0 & 0 & 1-\left(1-d_{f}\right)\left(1-d_{m}\right) v_{12} v_{21}\left(1-d_{s}\right) G\end{array}\right)$

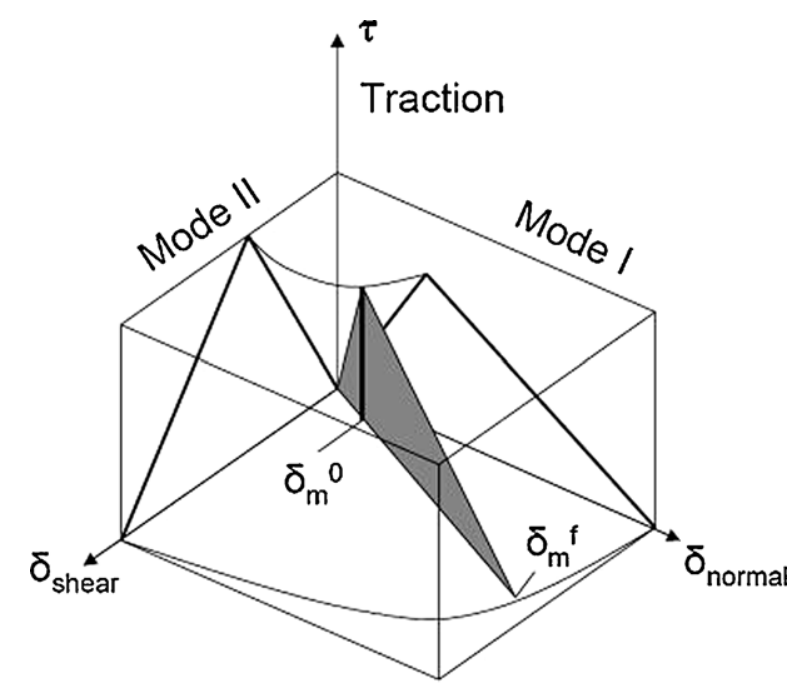

Fig. 3. Schematic of mixed mode softening law in interface elements after [20].

$E_{11}, E_{22}$, and $G_{12}$ are the longitudinal, transverse, and in-plane shear modulus of the undamaged orthotropic lamina. Detailed information regarding the mentioned in-ply damage model such as damage evolution laws or constitutive equations can be found in Refs. $[10,13,23]$.

\subsection{Interface model}

The cohesive zone approach, adopted in this work, makes use of interface finite elements incorporating a cohesive mixed mode damage model. In this section, a concise description of the interface element is given. The zero thickness eight node cohesive elements, implemented as a User Element in ABAQUS [23], are based on the constitutive model suggested by Dávila and co-workers [20], and the detailed mathematical formulation can be found in Refs. $[20,21]$.

The interface damage initiation is based on the quadratic interfacial traction interaction criterion as shown in Eq. (6):

$$
\left(\frac{\tau_{1}}{\tau_{1}^{0}}\right)^{2}+\left(\frac{\tau_{2}}{\tau_{2}^{0}}\right)^{2}+\left(\frac{\tau_{3}}{\tau_{3}^{0}}\right)^{2}=1
$$

$\tau_{3}, \tau_{1}, \tau_{2}$ are interface tractions in normal, 1 st and 2 nd shear directions, respectively and $\tau_{3}^{0}, \tau_{1}^{0}, \tau_{2}^{0}$ are the normal and shear elastic limits of the interface (Fig. 2). The interfacial damage propagation is based on constitutive interface tractions $\left(\tau_{3}, \tau_{1}, \tau_{2}\right)$ and relative displacements $\left(\delta_{3}, \delta_{1}, \delta_{2}\right)$ as

$$
\begin{aligned}
& {\left[\begin{array}{l}
\tau_{1} \\
\tau_{2} \\
\tau_{3}
\end{array}\right]=\left(\left(\begin{array}{lll}
(1-d) K & 0 & 0 \\
0 & (1-d) K & 0 \\
0 & 0 & (1-d) K
\end{array}\right)+\left[\begin{array}{l}
0 \\
0 \\
d K H\left(-\delta_{3}\right)
\end{array}\right]\right)\left[\begin{array}{l}
\delta_{1} \\
\delta_{2} \\
\delta_{3}
\end{array}\right]} \\
& H(x)=\left\{\begin{array}{ll}
0 & \mathrm{x}<0 \\
1 & \mathrm{x} \geq 0
\end{array}\right\} \\
& d=\frac{\delta_{m}^{f}\left(\delta_{m}-\delta_{m}^{0}\right)}{\delta_{m}\left(\delta_{m}^{f}-\delta_{m}^{0}\right)} \quad \delta_{m}=\sqrt{\delta_{1}^{2}+\delta_{2}^{2}+\delta_{3}^{2}}
\end{aligned}
$$




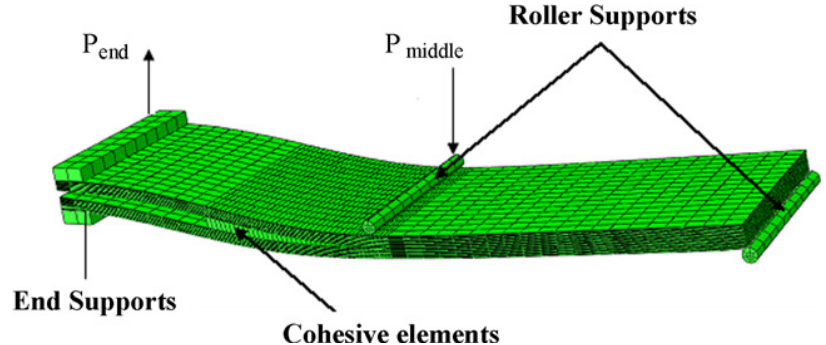

Fig. 4. Schematic view of the numerical model.

In Eq. (7), $K$ is the initial stiffness of the interface, and $d$ is the isotropic damage parameter. Prior to damage initiation, both faces of the interface element are bonded together with the high initial stiffness, $K$, and the value of $d$ is equal to zero. The relative displacement, corresponding to damage initiation $\left(\delta_{m}^{0}\right)$ in Fig. 3, is called the mixed mode opening displacement or the fictive crack tip. The initiated damage then starts evolving based on an energy based propagation criterion Benzeggagh and Kenane [31], which is described in Section 2.2. Meanwhile, the isotropic damage parameter degrades the interfacial tractions, as shown in Eq. (7), till the final separation point, $\delta_{m}^{f}$ in Fig. 3, is reached.

\subsection{Geometry of the numerical model and boundary conditions}

The numerical model was created using the object oriented ABAQUS Scripting Interface in ABAQUS [23] for further optimization and parametric studies. The laminate is made of 24 plies with different stacking sequences as defined in Section 2 (Table 1). Each lamina is defined using the in-built reinforced ply damage model with 8 node, reduced integration, continuum shell elements. The interface elements, implemented as User Element routine in ABAQUS [23], are placed in the mid-plane of the laminate to capture the delamination behaviour. Since the loading lever is not simulated, loading boundary conditions are applied directly to middle and end supports as shown in Fig. 4. Moreover, different mode mixities $G_{\text {shear }} / G_{T}$ ratios, are simulated by applying different displacement boundary conditions at mentioned supports. A schematic of the developed numerical model (orthotropic lamina and interface elements and applied boundary conditions) is shown in Fig. 4. Refined mesh is used in the areas near to the middle support and in the vicinity of the delamination plane to avoid excessive element deformations.

The input material parameters for each lamina are obtained from previous measurements [32]. Ultimate in-plane strength in fiber, transverse, and shear directions ( $X, Y$, and $S$, respectively) is determined from standard tension and compression coupon tests in fiber, matrix, and shear directions [33]. In order to obtain laminar fiber fracture $\left(G_{\text {fiber fracture }}\right)$ and matrix cracking $\left(G_{\text {matrix crack }}\right)$ strain energies, compact tension (CT) tests have been conducted, and the data were reduced based on the compliance method [34]. No standard data reduction scheme as well as test standards are currently available in literature for determination of intra-laminar toughness of composite materials. However, the compliance method, applied to $(\mathrm{CT})$ test specimens, seems to provide good approximations of the intra-laminar fracture toughness values. For determination of $\left(G_{\text {fiber fracture }}\right)(\mathrm{CT})$ experiments had been conducted on $[0 / 90]_{15}$ laminates where fibre breakage was the dominant failure mode and $\left(G_{\text {matrix crack }}\right)$ was measured using $[0]_{30}$ laminates as $(\mathrm{CT})$ specimens, where the mode of failure is generally matrix cracking. Interface properties $\left(G_{I c}, G_{I I c}\right.$, and $\left.\eta\right)$ are derived from own MMB experiments. $G_{I c}$ and $G_{\text {IIc }}$, are obtained from pure mode I (Double Cantilever Beam) and pure mode II (End Notch Flexure) test results, using the Corrected Beam Theory data reduction scheme explained in Eq. (1). $\eta$ is the parameter, which best fits the Benzeggagh and Kenane criterion [31] when the total fracture toughness $G_{c}$, obtained through Eq. (1) for each mode mixity, is plotted versus corresponding mode mix, $G_{\text {shear }} / G_{T}$, values (illustrated in Fig. 5a). Normal and shear interfacial strengths are estimated to be $75-80 \%$ of resin strengths [35]. The initial stiffness, $K$, is approximated to be $10^{7}$. Based on the work of Allix and Blanchard [18], this value is close to the ratio of the adjacent ply stiffness in thickness direction, $E_{33}$, and the thickness of the interface, $t$, which is assumed to be $0.001 \mathrm{~mm}\left(K=E_{33} / t\right)$. The required input parameters for the numerical model are summarized in Table 2.

\section{Results and discussion}

\subsection{Mechanical test results and numerical simulations}

Having determined the required material parameters for the numerical model, the load versus load point displacement responses, obtained numerically and experimentally for three different mode mixities (30\%, 50\%, and $80 \%$ ) on unidirectional laminates, are compared together, and the results are presented in Fig. 5b. According to the graph, good agreement is achieved between experiments and numerical simulations. The simulated curves are based on the specified Benzeggagh and Kenane (B-K) propagation criterion, which is determined by a fit curve trough all available experimental data, as shown in Fig. 5a. The results of individual experiments might not exactly coincide with the prediction of the simulation, since some uncertainties in the specified material parameters might exist, which in turn might affect the simulation results.

Table 2 lists about 20 parameters as mechanical properties of lamina and interface that all by necessity contain some uncertainty. The deviation occurring in the FE calculation of maximum failure load, for a specified displacement, as a function of probable uncertainties in some material parameters is shown graphically in Fig. 5c. The specified parameters for the lamina are all obtained as a result of conducted experiments, such as coupon tension and compression tests and (CT) experiments as an average of at least

Table 2

Mechanical properties of lamina ( $t$ : tension, $c$ : compression, ${ }^{*} i s$ : in situ) and the $0 / 0$ (UD) interface.

\begin{tabular}{|c|c|c|c|c|c|}
\hline$E_{11}(\mathrm{MPa})$ & $E_{22}(\mathrm{MPa})$ & $v_{12}$ & $G_{12}(\mathrm{MPa})$ & $G_{23}(\mathrm{MPa})$ & $G_{\text {fiber fracture }}\left(\mathrm{mJ} / \mathrm{mm}^{2}\right)$ \\
\hline \multicolumn{6}{|c|}{ Mechanical properties of lamina ( $t$ : tension, $c$ : compression, is: in situ) [32] } \\
\hline 138000 & 10500 & 0.3 & 6300 & 3500 & 3.45 \\
\hline$X_{t}(\mathrm{MPa})$ & $X_{c}(\mathrm{MPa})$ & $Y_{t}(\mathrm{MPa}), Y_{t}^{i s}(\mathrm{MPa})^{*}$ & $Y_{c}(\mathrm{MPa})$ & $S(\mathrm{MPa}), S^{i s}(\mathrm{MPa})^{*}$ & $G_{\text {matrix crack }}\left(\mathrm{mJ} / \mathrm{mm}^{2}\right)$ \\
\hline 2070 & 1360 & $86,155^{*}$ & 196 & $147,205.8^{*}$ & 1.2 \\
\hline$\tau_{n}^{0}(\mathrm{MPa})$ & $\tau_{s}^{0}=\tau_{t}^{0}(\mathrm{MPa})$ & $K(\mathrm{MPa})$ & $G_{\mathrm{Ic}}\left(\mathrm{mJ} / \mathrm{mm}^{2}\right)$ & $G_{\text {IIc }}\left(\mathrm{mJ} / \mathrm{mm}^{2}\right)$ & $\eta$ \\
\hline \multicolumn{6}{|c|}{ Mechanical properties of interface } \\
\hline 75.45 & 80.42 & $10^{7}$ & 0.97 & 1.72 & 2.3 \\
\hline
\end{tabular}



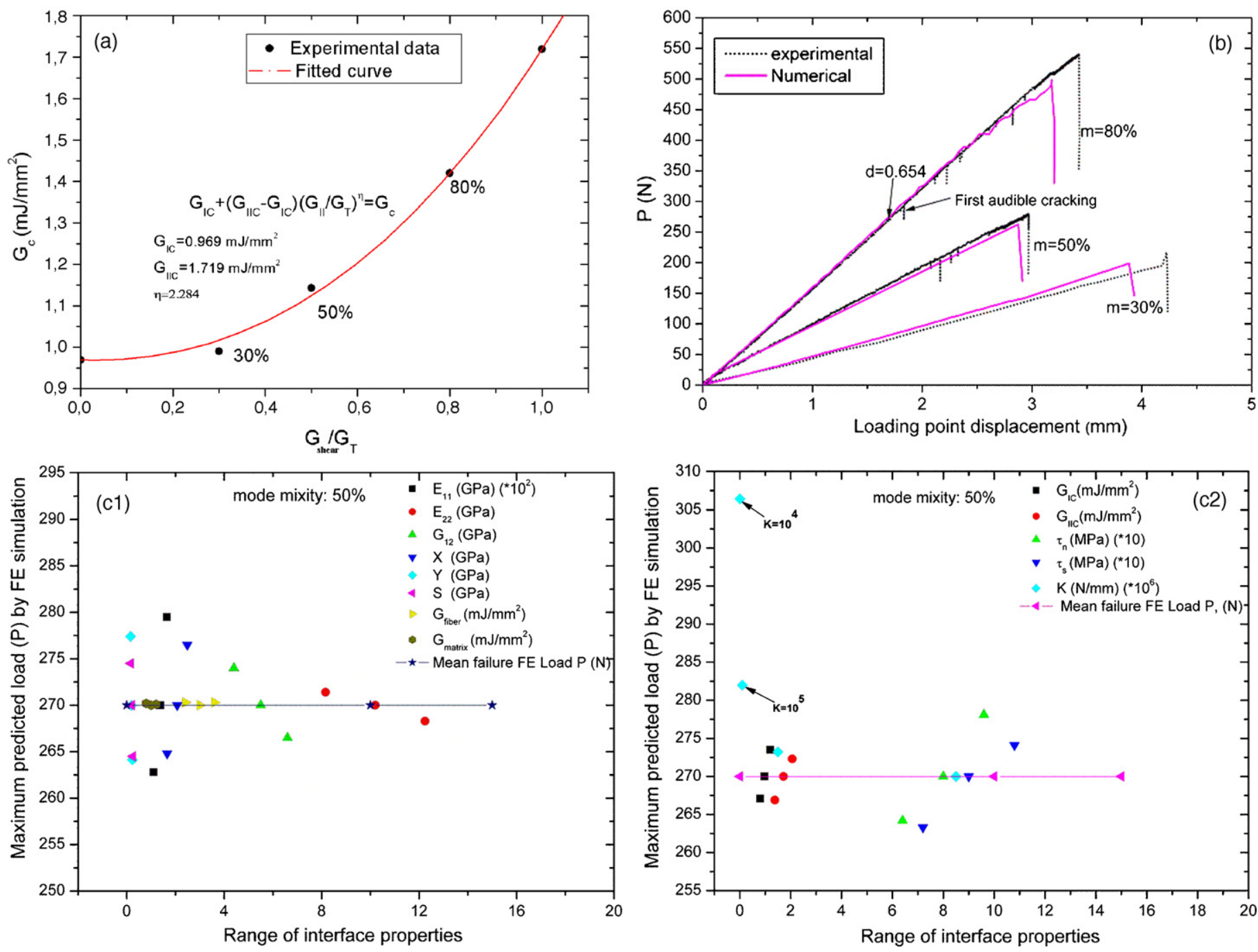

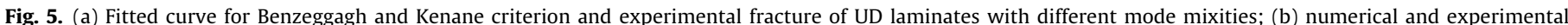

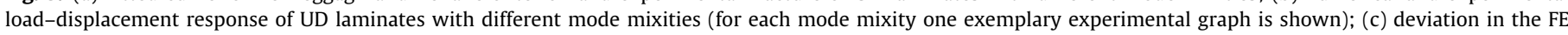

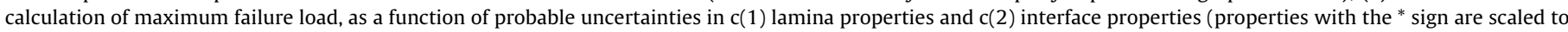
fit in the defined range. Ex: $E_{11}$ value in c(1) must be multiplied by $10^{2}$ to reach the value of $138 \mathrm{GPa}$ listed in Table 2 ).

five specimens per test. Hence, the degree of uncertainty in lamina parameters is expected to be very low. Assuming $20 \%$ uncertainty range in used laminar properties (listed in Table 2), causes maximum $3.70 \%$ deviation in the estimated failure load value by $\mathrm{FE}$ simulations (cf. Fig. $5 c(1)$ ). Meanwhile, some specified interface properties such as $G_{I c}, G_{I I c}$ and $\eta$ are obtained through MMB experiments, and some of them like the initial stiffness of the interface element $(K)$ or initial interfacial strengths, $\tau_{3}^{0}$ and $\tau_{1}^{0}$, are estimated based on mathematical approximations. The deviation in calculated failure load by FE simulation is about $2.7 \%$ for mathematically approximated $\tau_{3}^{0}$ and $\tau_{1}^{0}$ while it falls below $1.9 \%$ for $G_{\text {Ic }}$ and $G_{\text {IIc }}$ for the same uncertainty range of $20 \%$ (cf. Fig. $5 c(2)$ ). Final point is the effect of initial interfacial stiffness, $K$ on the FE prediction of the failure load. Fig. $5 c(2)$ indicates that $K$ must be chosen at least as large as $10^{5}$, to avoid higher values of deviations about $13.5 \%$ in $\mathrm{FE}$ calculations of failure load.

With increasing mode mixity, the critical applied load required for structural failure is also increased, as a result of increasing interlaminar shear forces in between crack surfaces. In the numerical simulations, the maximum interface damage parameter of all cohesive interface elements, $d$, can be tracked throughout the loading history in the specified cohesive zone. It is basically observed that this parameter starts growing very close to the first audible cracking point, shown in Fig. 5b, which also stands for a very good damage prediction of the working numerical model. Throughout the MMB experiments, for the highest mode mixity ( $80 \%$ ) the crack propagation rate is higher and more instability is observed in the last stages of crack propagation. This in turn can trigger the more often occurrence of minor load drops in the load-displacement curve of $80 \%$ mode mixity. Meanwhile, microstructure investigations of the UD specimen under $80 \%$ mode mixity, explained in detail in Section 4.2 , represent more often formation and occurrence of shear cusps and coalescence of microcracks. These microscopically observed phenomena make the fracture surface rougher, re-propagation of the crack harder and more unstable, which can lead to more frequent occurrence of minor load drops. In a numerical point of view, minor load drops, in Fig. 5b, could be correspondent to the growth of the damage parameter, $d$, and this growth follows a faster trend closer to the ultimate load.

Fig. 6 shows the load-displacement response of MD and UD laminates subjected to MMB. It is basically observed that fiber angle orientation and stacking sequences have a global effect on load-displacement response. The in situ effects in MD laminates are characterized by higher transverse tensile and shear strengths of a ply when it is constrained by plies with different fiber orientations in a laminate, compared with the strength of the same ply in 


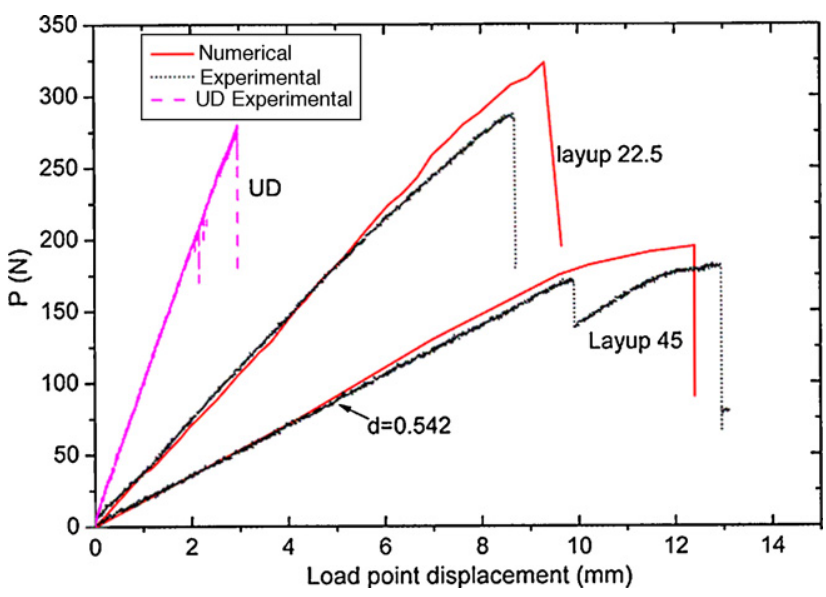

Fig. 6. Numerical and experimental load-displacement response of MD and UD laminates with $50 \%$ mode mixity.

a unidirectional laminate [36-37]. In this work, the in situ tensile and shear strengths for MD laminates are estimated based on the approach suggested in Ref. [37] and replace the ones used for the UD layup (Table 2). After specifying the ply and interface material parameters, obtained experimentally, FE simulations and experimental results for each layup can be compared together (Fig. 6). The results belong to $50 \%$ mode mixity, and a good agreement is achieved in load-displacement response. The conducted experiments (Table 3 ), prove that the mixed mode fracture toughness of
Table 3

Comparison of experimentally determined fracture toughness results for different MD laminates $(m=50 \%)$

\begin{tabular}{ll}
\hline Layup name & $\begin{array}{l}\text { Experimental mixed mode fracture } \\
\text { toughness } G_{c}\left(\mathrm{~mJ} / \mathrm{mm}^{2}\right)\end{array}$ \\
\hline Layup 22.5 & 1.64 \\
Layup 45 & 1.68 \\
Layup UD & 1.15 \\
\hline
\end{tabular}

MD laminates is considerably greater than UD ones with about $45 \%$ increase observed in both of the mentioned layups.

\subsection{Crack paths and fracture surfaces}

First point of interest in MD laminates subjected to MMB are the curved crack fronts (Fig. 7a), observed for example in layup 45 , which is considered as an extrinsic toughening mechanism and will improve the fracture toughness and make it difficult for the crack to re-propagate [7]. In contrast, in UD laminates the crack front remains rather straight. Second point is deviation of the crack from the delamination mid-plane to the adjacent disoriented ply observed at later stages of the experiment (Fig. 7b). This will also contribute to some amount of energy absorption through in-ply damage in the adjacent layer, which again leads to an increase in the total mixed mode fracture toughness [38].

Mixed mode delamination fracture surfaces produced under different mode mixities and different stacking sequences are investigated by SEM. Fig. 8 displays typical fracture surfaces obtained on UD laminates under $30 \%$ and $80 \%$ mode mixity. (a)

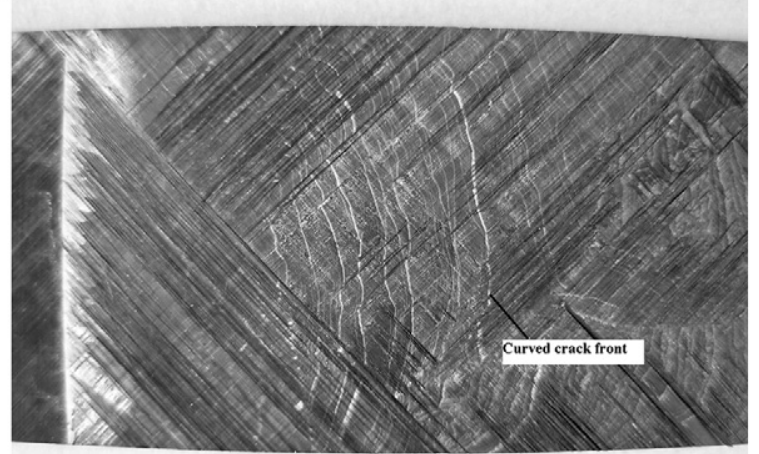

(b)

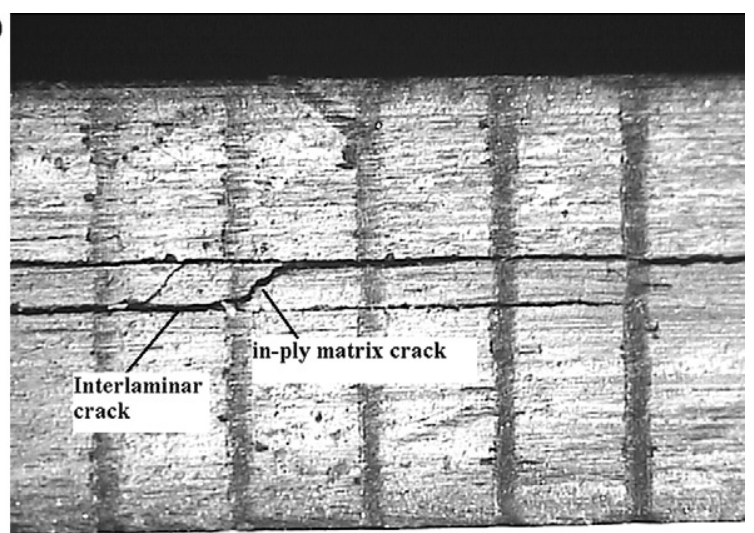

Fig. 7. Extrinsic toughening mechanisms in MD laminates: (a) curved crack fronts and (b) observed in-ply matrix damage.

(a)

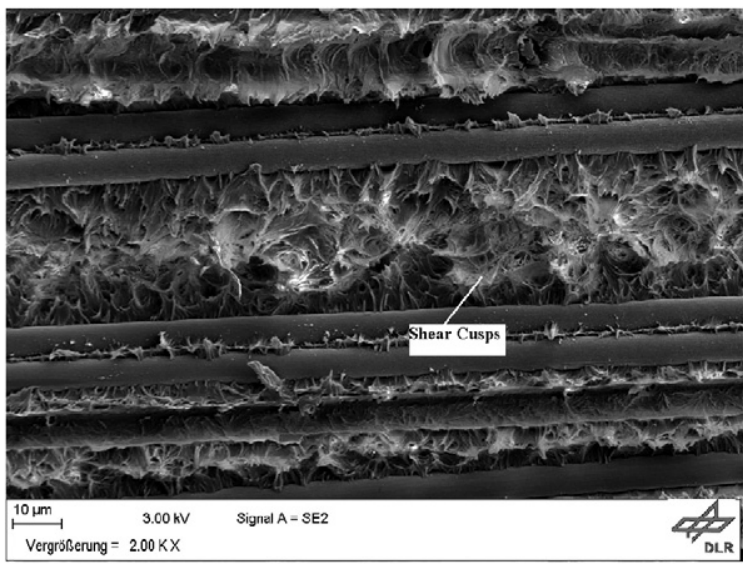

(b)

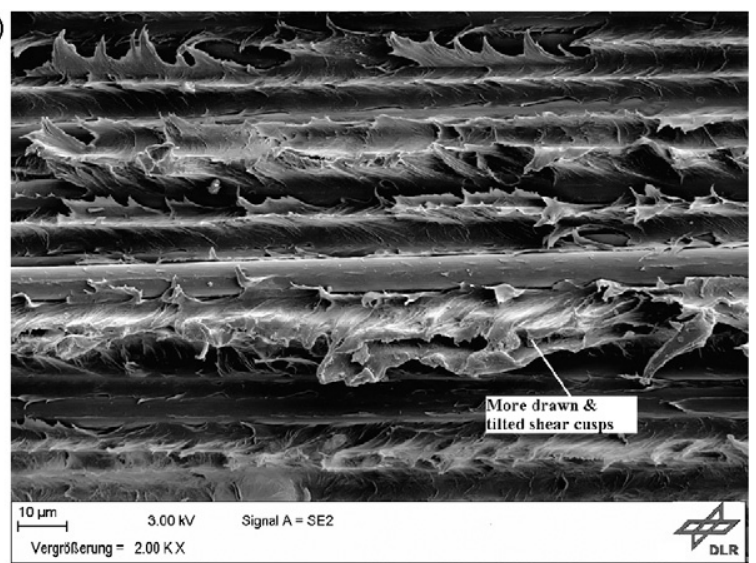

Fig. 8. Fracture surfaces of $0 / 0$ (UD) interface under $30 \%$ (a) and $80 \%$ (b) mode mixity. 

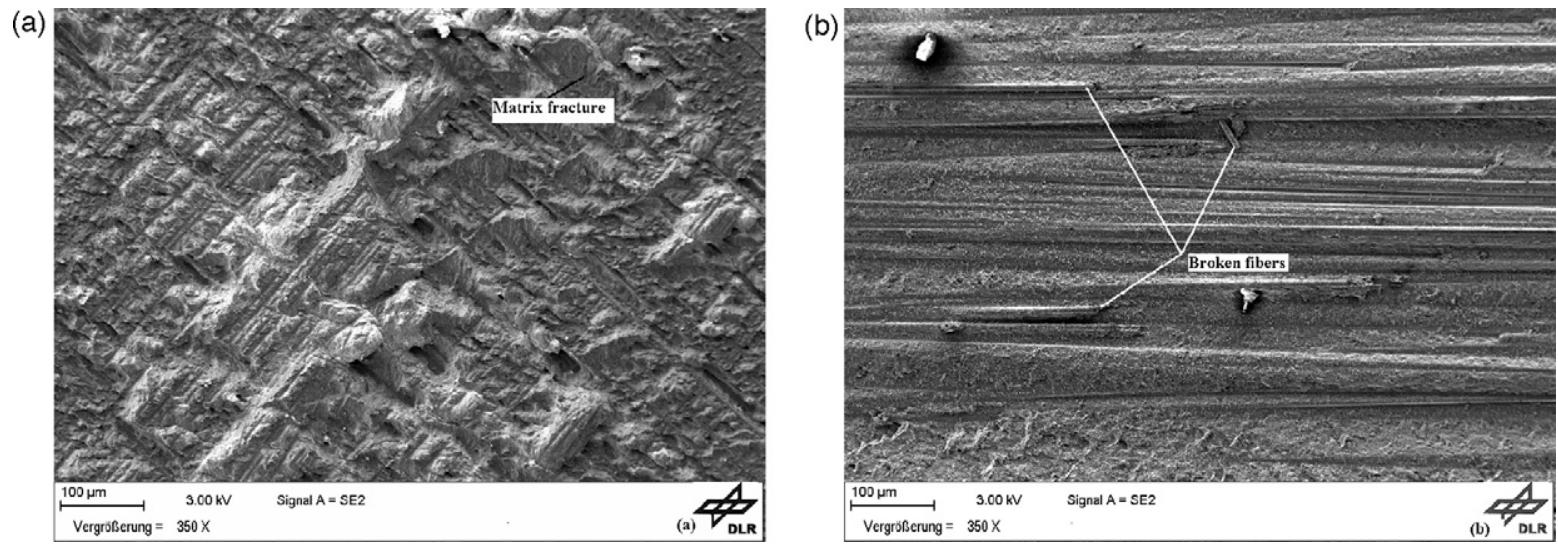

Fig. 9. Fracture surfaces of (a) layup 45 interface and (b) UD interface, under 50\% mode mixity.

The inter-laminar fracture surfaces obtained under $80 \%$ mode mixity exhibited a rougher topography than the ones obtained under $30 \%$ mode mixity. In Fig. $8 a$ and b, it can be seen that the roughness is due to more dense and upwardly drawn shear cusps, which are caused by the extensive yielding (ductility) of the matrix. The primary difference between the fracture surfaces at different mixed mode ratios can be seen in the orientation of the cusps. The $30 \%$ fracture surface (Fig. 8a) has a few number of cusps already with the tendency to be drawn and tilted due to existing mode II action, and larger amount of mode II (80\%) draws and tilts the cusps more and more due to the higher amounts of shearing action (Fig. 8b).

Similar observations about the dependency of appearing roughness of delamination crack surfaces on the mode mixity have been reported by Reeder and Crews [28], who compared inter-laminar fracture surfaces produced under various mode mixities. The presence of the cusps, which are more expressed under conditions of higher mode II loading proportion, suggests inter-laminar shear as the primary load source behind their formation. Morris [39] suggested that the formation of shear cusps is correlated with flexural loading associated with local bending of the fracture surface just behind the crack tip. However, according to Smith and Groove [40] their formation occurs by coalescence of numerous microcracks inclined at an angle to the plane of applied shear. Denser appearance of upwardly drawn cusps in $80 \%$ mode mix (Fig. 8b), which has larger amount of contributing inter-laminar shear, supports the idea presented in Ref. [28]. Additionally, confirming the idea of Smith and Groove [40], formation and coalescence of microcracks corresponding to the growth of the damage parameter, $d$ (Fig. 5b) is also treated as a potential source responsible for formation of these shear cusps.

It is also worth to note, that the fracture toughness of the here tested material with the thermoplastic matrix (PEEK) is larger than that of the epoxies tested in literature [28]. This larger fracture toughness can be referred to the larger strain to failure created by the yielding of the thermoplastic PEEK.

One of the main differences between the fracture surface of the PEEK composite studied here and epoxy is that all the PEEK fracture surfaces ranging from pure mode I to pure mode II have cusps caused by the higher amount of matrix plastification. In contrast, in an epoxy composite, referred in literature, the fracture surfaces of lower modes are completely flat indicating brittle cleavage fracture. Another difference is the appearance of hackles with higher mode mixity in an epoxy composite, whereas no hackle formation is observed in PEEK. These differences between fracture surfaces indicate different failure mechanisms for these two classes of polymer matrix composites.
MMB delamination between $+45 /-45$ ply interfaces exhibited some distinct differences in fracture morphology compared to $0 / 0$ interface under the same mode mixity (Fig. 9).

The overall fracture surfaces of the $+45 /-45$ interface exhibited more cohesive matrix fracture than the $0 / 0$ interface as illustrated in Fig. 9a. Longitudinally oriented rows of fan-shaped matrix fracture areas, oriented with a slight tilt to the overall fracture surface, are observed in the related SEM micrograph (Fig. 9a). Since the failure of $+45 /-45$ interface is dominated by matrix fracture, the maximum load to failure is not as large as for the $0 / 0$ interface, where the failure exhibits broken fibers, which have been bridging the crack (Fig. 9b). According to [40], the appearance of broken fibres ahead of the crack tip in the fracture zone has a direct effect on rising the fracture load in laminated composites. This fact can also be verified by comparing the mixed mode failure load levels of layups UD and 45 in Fig. 6.

\section{Summary and conclusion}

This paper studies the effect of fiber orientation and different stacking sequences on mixed mode delamination failure in UD and MD laminates following experimental and numerical approaches. The load-displacement response of MD composites subjected to MMB is strongly dependent on the stacking sequence of the laminate. Inter-laminar mixed mode fracture toughness of MD laminates increases considerably compared to UD ones as a result of some observed extrinsic toughening mechanisms such as appearance of curved crack fronts and intra-laminar energy absorption. SEM micrographs also provide detailed information on the nature of mixed mode fracture under different mode mixities and give further clarifications on the probable micro-mechanical origins of observed different fracture energies in various layups. The numerical model based on using damageable plies and interface elements has been validated successfully through comparison with experimental results for different mode mixities and various layups. It is also significant that all interface parameters must be specified correctly in order to obtain solution convergence.

\section{Acknowledgements}

The authors acknowledge the effort of the technicians and coworkers in the institute of Structure and Design of DLR, Stuttgart for producing the CFRP laminates. The authors also thank the mechanical testing group in the institute of material research, Cologne, specially Janine Schneider for conducting the experiments. 


\section{References}

[1] J. Andersons, M. Konig, Compos. Sci. Technol. 64 (2004) 2139-2152.

[2] A. Quispitupa, C. Berggreen, L.A. Carlsson, Eng. Fract. Mech. 59 (2009) 594-613.

[3] N.S. Choi, A.J. Kinloch, J.G. Williams, J. Compos. Mater. 33 (1999) 73-100.

[4] M.R. Piggot, W. Zhang, ESIS: Fract. Polym. Compos. Adhes. II 32 (2003) 445454.

[5] A.B. de Morais, A.B. Pereira, Compos. Sci. Technol. 66 (2006) 1889-1895.

[6] B.K. Raghu Prasad, D.V.T.G. Pavan Kumar, Thin-Wall. Struct. 46 (2008) 676688.

[7] T.L. Anderson, Fracture Mechanics: Fundamentals and Applications, CRC Press Inc., 2005.

[8] B.D. Davidson, R.D. Bialaszewski, S.S. Sainath, Compos. Sci. Technol. 66 (2006) 1479-1496.

[9] P. Ladeveze, E. Le Dantec, Compos. Sci. Technol. 43 (1992) 257-267.

[10] A. Matzenmiller, J. Lubliner, R.L. Taylor, Mech. Mater. 20 (1995) 125-152.

[11] C.T. Herakovich, Mechanics of Fibrous Composites, Wiley, New York, 1998

[12] P. Ladeveze, L. Guitard, L. Champaney, X. Aubard, Comp. Meth. Appl. Mech. Eng. 183 (2000) 109-122.

[13] Z. Hashin, J. Appl. Mech. 20 (1981) 329-334.

[14] J.R. Rice, J. Appl. Mech. 35 (1968) 379-386.

[15] E.F. Rybicki, M.F. Kanninen, Eng. Fract. Mech. 9 (1977) 931.

[16] D.S. Dugdale, Nonlinear Dynam. 8 (1960) 100-104

[17] A. Hillerborg, M. Modeer, P.E. Petersson, Cement Concrete Res. 6 (1976) $773-782$.

[18] O. Allix, L. Blanchard, Compos. Sci. Technol. 66 (2006) 731-744.

[19] S. Li, M.D. Thouless, A.M. Waas, J.A. Schroeder, P.D. Zavattieri, Eng. Fract. Mech. 73 (2006) 64-78.
[20] A. Turon, C.G. Dávila, P.P. Camanho, J. Costa, Eng. Fract. Mech. 74 (2007) 1665-1682.

[21] Z.-H. Jin, C.T. Sun, Int. J. Solids Struct. 43 (2006) 1047-1060.

[22] J.H. Crews, J.R. Reeder, NASA TM 100662 (1988).

[23] Hibbitt, Karlsson, Sorensen, ABAQUS 6.6 User's Manuals, Pawtucket, USA, 1996.

[24] R.M. Jones, Mechanics of Composite Materials, New York, McGraw-Hill, 1975.

[25] Test Method D6671-01, Standard Test Method for Mixed Mode I/Mode II Interlaminar Fracture Toughness of Unidirectional Fiber Reinforced Polymer Matrix Composites, ASTM, PA, USA, 2002.

[26] J.G. Williams, J. Strain Anal. 24 (1989) 207-214.

[27] J.R. Reeder, J.H. Crews, NASA TM 102777 (1991).

[28] A.B. Pereira, A.B. de Morais, Compos. Sci. Technol. 39 (2008) 322-333.

[29] J. Wang, P. Qioa, Eng. Fract. Mech. 71 (2004) 219-231.

[30] F. Ducept, P. Davies, D. Gamby, Int. J. Adhes. Adhes. 20 (2000) 233-244.

[31] M.L. Benzeggagh, M. Kenane, Compos. Sci. Technol. 56 (1996) 439-449.

[32] D. Kohlgruber, Internal Report: Mechanical properties of PEEK/AS4. Source: CYTEC/DLR, Institute of Structures and Design, German Aerospace centre-DLR, Stuttgart, 1997.

[33] Test Method D 3039/D 3039M, Standard Test Method for Tensile Properties of Polymer Matrix Composites, ASTM, PA, USA (2000).

[34] J.M. Slepetz, Fracture of Composite Compact Tension Specimens. ASTM STP 593, ASTM, 1975.

[35] K. Liu, M.R. Piggott, Polym. Eng. Sci. 38 (1998) 60-68.

[36] G.J. Dvorak, N. Laws, J. Compos. Mater. 21 (1987) 309-329.

[37] C.G. Davila, P.P. Camanho, C.A. Rose, J. Compos. Mater. 39 (2005) 323-345.

[38] A.J. Brunner, P. Flueler, Eng. Fract. Mech. 72 (2005) 899-908.

[39] G.E. Morris, ASTM STP 696 (1979) 274-297.

[40] B.W. Smith, R.A. Grove, ASTM STP 948 (1987) 154-173. 